\title{
Study on Catalytic Conversion of Cellulose to 5-Hydroxymethyl Furfural by Directional Degradation in Deep Eutectic Solvents
}

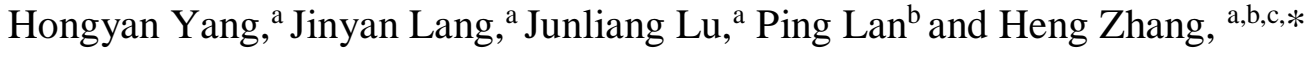 \\ The direct conversion of microcrystalline cellulose (MCC) degrading into \\ 5-hydroxymethyl furfural (5-HMF) was studied using four synthetic deep \\ eutectic solvents (DESs) as solvents and choosing four metal chlorides as \\ catalysts. The factors that affected the yields of the products, such as the \\ type of DESs, the type and dosage of catalysts, the ratio of DESs to MCC, \\ and reaction temperature were researched. It was found that the DES \\ synthesized from oxalic acid and choline chloride (O-DES) and $\mathrm{SnCl}_{4}$ \\ showed the better performance. The highest 5-HMF and glucose yields of \\ $11.0 \%$ and $22.0 \%$ were obtained, respectively, for a reaction carried out \\ at $160{ }^{\circ} \mathrm{C}$ for $90 \mathrm{~min}$ in O-DES (the ratio of it to MCC was 25:1) using 1.42 \\ wt\% $\mathrm{SnCl}_{4}$ as the catalyst. As a new type of dissolution and catalysis \\ system, the DESs were not only cheaper but also easy to obtain. Most \\ importantly, the realization of the concept of green chemistry was achieved \\ in this study.
}

Keywords: 5-HMF; DESs; Glucose; MCC

Contact information: a: College of Marine Science and Biological Engineering, Qingdao University of Science \& Technology, Qingdao, Shandong 266042, P.R. China; b: Guangxi Key Laboratory of Polysaccharide Materials and Modification, School of Chemistry and Chemical Engineering, Guangxi University for Nationalities, Nanning, 530008, P.R. China; c: Key Laboratory of Biomass Chemical Engineering of Ministry of Education, Zhejiang University, Hangzhou 310027, China;

*Corresponding authors: hgzhang@ sina.com

\section{INTRODUCTION}

Cellulosic biomass is one of the most abundant renewable plant resources available on earth. The annual global production of lignocellulosic biomass is over 340 billion tons (Ge et al. 2016). It is an important aspect in the development of green- and circulareconomies that cellulose can be converted directly into platform compounds and fine chemicals. Hydroxymethyl furfural (5-HMF) is an important intermediate compound, which could serve as a renewable biomass resource to prepare liquid fuel, a series of chemicals, and synthesize varieties of biofuels (Feng et al. 2010), fine chemicals (Bozell and Petersen 2010), and polymers (Tong et al. 2010). For example, the addition of solid bases in the presence of certain reagents followed by condensation dehydration could form a series of liquid alkanes for the replacement of petroleum fuels (Huang et al. 2012; Hronec et al. 2014). Furan-dicarboxylic acid, which can be obtained by further oxidation, was used as the initial raw material for the synthesis of polyester materials (Casanova and Iborra 2010; Villa et al. 2013). It is an ideal biomass platform compound (Román-Leshkov et al. 2007) to reduce or avoid the consumption of fossil resources and has been listed as one of twelve high value-added biomass derivatives by the U.S. Department of Energy.

Deep eutectic solvents (DESs), which are mixtures composed of hydrogen-bond acceptors and hydrogen-bond donors having a certain molar ratio that can bond with each 
other, were first proposed by Abbott et al. (2003). The main preparation methods of DESs involve heating (Durand et al. 2013), grinding (Florindo et al. 2014), and rotary evaporation (Dai et al. 2013; Mjalli et al. 2012). Their melting points are lower than that of any single component. The earliest application areas of DESs were metal electrodeposition and electropolishing (Abbott and Mckenzie 2006). Subsequently, DESs as reaction media have been rapidly applied in the fields of organic compound synthesis (Mota-Morales et al. 2013), biocatalysis (Zhao et al. 2011), polymer production (Ramesh et al. 2013), electrochemistry (Bahadori et al. 2013), preparation of nanomaterials (Huang et al. 2013), separation processes (Guo et al. 2013), biomedicine (Sanchez-Leija et al. 2014), and extraction of natural active products (Cui et al. 2015; Wei et al. 2015). Later, Ilgen et al. (2009) and Han et al. (2009) used choline chloride (ChoCl)-based DESs as solvent and fructose as raw materials to prepare 5-HMF, which yielded 5-HMF over 30\% and 90\%, respectively. Matsumiya (2015) used DES synthesized from citric acid dihydrocholine and glycolic acid as a solvent system and $\mathrm{B}(\mathrm{OH})_{3}$ as catalyst to obtain 5HMF from glucose with a maximum yield of $42 \%$. Liu et al. (2013) showed that $90 \%$ yield of HMF could be obtained from fructose in DES mixture formed by citric acid and ChoCl. Therefore, it was feasible to prepare 5-HMF from glucose or fructose in DESs.

Considering the economic factors, it would be ideal to use cellulose with lower price as raw material. However, cellulose is not easy to degrade because of higher crystallinity, so this will be a greater challenge. Liu et al. (2013) degraded microcrystalline cellulose (MCC) pretreated with $\mathrm{FeCl}_{3}$ and $\mathrm{AlCl}_{3}$ at $200{ }^{\circ} \mathrm{C}$ in the two-phase system of $\mathrm{H}_{2} \mathrm{O}-\mathrm{ChoCl} / \mathrm{MIBK}$ to obtain 5-HMF with maximum yield of $27 \%$, and they degraded MCC pretreated with ionic liquids (at this time, the degree of polymerization of MCC was very low) to obtain 5-HMF with maximum yield of $49 \%$. This demonstrates that it is feasible to obtain 5-HMF from MCC using DESs as solvent. In this paper, on the basis of previous studies (Matsumiya 2015; Zuo et al. 2017), several types of DESs were synthesized as dissolving systems, and metal chloride as catalyst were used to degrade MCC with higher degree of polymerization that is closer to industrial cellulose or MCC from dissolved pulp (degree of polymerization degree of 251). The feasibility of preparing 5-HMF by degrading MCC in DESs system was systematically studied in this work, which could provide the theoretical basis and data support for large-scale production in the future.

\section{EXPERIMENTAL}

\section{Materials}

Glucose was purchased from Henan Lianchuang Chemical Co. Ltd, Jiyuan, China. Chromium chloride (99\%), choline chloride (99\%), citric acid (98\%), and glycerol (98\%) were obtained from Shandong Xiya, Linyi, China. Urea (97\%) and oxalic acid (98\%) were purchased from Tianjin Damao, Tianjin, China. The MCC (polymerization degree of 251) was obtained from Chengdu Kelon, Tianjin, China. The 5-hydroxymethyl furfural (HPLC grade, $99.9 \%$ ) was obtained from Shandong Xiya, Linyi, China. Copper chloride (98\%), ferric chloride (99\%), and tin chloride (98\%) were purchased from Tianjin Beichen Fangzheng Reagent Factory, Tianjin, China.

\section{Preparation of 5-HMF and Glucose from Cellulose Degraded by DESs}

The required reagents were dried in a vacuum drying chamber at $50{ }^{\circ} \mathrm{C}$ for $48 \mathrm{~h}$. Hydrogen bond donors and hydrogen bond acceptors (molar ratio=2:1) were added to a 
four-neck flask, stirred and heated under nitrogen in an oil bath at a specified temperatures. When a fully homogeneous transparent liquid was obtained, the reaction was stopped, and transferred to a plug bottle. Then, the prepared DESs were dried for $48 \mathrm{~h}$ in a vacuum dryer (vacuum dryer, DHG, Ta Company, USA) at $70{ }^{\circ} \mathrm{C}$ and stored in a desiccator.

The DESs synthesized with choline chloride as hydrogen bond acceptor and oxalic acid, citric acid, urea, and glycerol as hydrogen bond donors were abbreviated as O-DES, C-DES, U-DES, and G-DES, respectively.

Prepared DESs were added into a four-neck flask under $\mathrm{N}_{2}$ and heated to $100{ }^{\circ} \mathrm{C}$. The reaction was adjusted to the set temperature until DESs were dissolved completely, and then a certain amount of MCC was added. The solvent and MCC were mixed sufficiently. Then, the catalysts were added to start the reaction. A certain amount of the reaction solution was taken up with a pipette every half hour and diluted by 20 to 50 times before measuring the absorbance with a UV spectrophotometer (Zhang et al. 2018). The yields of products were calculated according to the test methods of Hou et al. (2017).

\section{Calculation of Product Yields}

The calculation for glucose yield was as follows,

$$
Y_{\mathrm{G}}=\frac{C_{\mathrm{G}} \times V}{m} \times 100 \%
$$

where $C_{\mathrm{G}}$ represents glucose concentration in $\mathrm{mg} / \mathrm{mL} ; V$ indicates the volume of the reaction solution in $\mathrm{mL}$; and $m$ is the initial amount of MCC in (mg).

The calculation for 5-HMF yield was as follows,

$$
Y_{5-\mathrm{HMF}}=\frac{C_{5}-\mathrm{HMF} \times 180 \mathrm{~V}}{126 \mathrm{~m}} \times 100 \%
$$

where $C_{5-\mathrm{HMF}}$ represents the concentration of $5-\mathrm{HMF}$ in $\mathrm{mg} / \mathrm{mL}$.

\section{RESULTS AND DISCUSSION}

\section{Effect of DESs Type on Product Yield}

An MCC:DESs ratio of 1:25 (mass ratio) was used. The yields of products of the reactions were calculated after $1.5 \mathrm{~h}, 2 \mathrm{~h}, 3 \mathrm{~h}$, and $12 \mathrm{~h}$ at $170{ }^{\circ} \mathrm{C}$, respectively. Table 1 shows the yields of 5-HMF and glucose from the degradation of cellulose varied when different types of DESs were used.

Table 1. Effect of DESs Types on Product Yields

\begin{tabular}{|c|c|c|c|c|}
\hline Products Yields & \multicolumn{4}{|c|}{ Types of DESs } \\
\cline { 2 - 5 }$(\%)$ & G-DES & U-DES & C-DES & O-DES \\
\hline 5 -HMF & 0.59 & 0.26 & 1.8 & 8.6 \\
\hline Glucose & 7.9 & 3.8 & 10.26 & 16.6 \\
\hline
\end{tabular}

Among the four types of DESs, the yields of 5-HMF and glucose were the highest in O-DES, achieving $8.6 \%$ and $16.6 \%$ yields, respectively, followed by C-DES, and the lowest yields were obtained in U-DES. The viscosity of DESs had a great influence on the dissolution of MCC. A lower viscosity was more advantageous to the movement of ions, and the MCC was dissolved easily. However, the viscosity of U-DES was much higher than that of O-DES. When dissolving MCC, the reaction mixture was in a viscous state, 
which resulted in the reactants sticking to the reactor wall, decreasing the contact between solvent molecules and MCC. The 5-HMF produced in the reaction was encapsulated by solvent, causing the reunion of 5-HMF with solvent molecules that increased the side reactions. Therefore, the dissolution effect of MCC by U-DES and the yields of products were poorer than that of three other DESs. In O-DES, the yield of glucose was $16.6 \%$, while that of 5-HMF was only $8.6 \%$. The 5-HMF was unstable in O-DES and did not extract from the solution in time, which led to side reactions. In the initial stage of reaction, the color of the reaction solution was transparent and colloidal. With increasing reaction time, the color of the solution changed to black (Wang et al. 2019). It was presumed that this was due to the instability of 5-HMF and this resulted in some side reactions as well.

The results showed that O-DES exhibited the highest solubility for MCC compared with the other three DESs, which was conducive to the production of 5-HMF. Therefore, O-DES was chosen as the next reaction solvent.

\section{Effect of the Type of Catalyst Used on Product Yields}

The MCC was added to O-DES solvent at the ratio of 1:25 (mass ratio). Then four metal chlorides were tested as catalysts, respectively. The yields of 5-HMF and glucose were explored at the reaction temperature of $150{ }^{\circ} \mathrm{C}$ for $1.5 \mathrm{~h}$.

Table 2. Effect of Catalysts Types on Product Yields

\begin{tabular}{|c|c|c|c|c|}
\hline \multirow{2}{*}{$\begin{array}{c}\text { Products Yields } \\
(\%)\end{array}$} & \multicolumn{4}{|c|}{ Types of Catalysts } \\
\cline { 2 - 5 } & $\mathrm{SnCl}_{4}$ & $\mathrm{CrCl}_{3}$ & $\mathrm{CuCl}_{2}$ & $\mathrm{FeCl}_{3}$ \\
\hline 5-HMF & 11.06 & 10.79 & 6.91 & 7.60 \\
\hline Glucose & 22.70 & 20.10 & 17.23 & 19.20 \\
\hline
\end{tabular}

As shown in Table 2, the yields of 5-HMF and glucose varied depending on the types of catalysts used. Among the four catalysts, $\mathrm{SnCl}_{4}$ had the best catalytic effect, and the maximum yields of 5-HMF and glucose were $11.06 \%$ and $22.7 \%$, respectively. When $\mathrm{CrCl}_{3}$ was used as the catalyst, the yields of 5-HMF and glucose were $10.79 \%$ and $20.1 \%$, respectively. The catalytic effect of $\mathrm{CrCl}_{3}$ was similar to that of $\mathrm{SnCl}_{4}$. The worst catalyst was $\mathrm{CuCl}_{2}$. Four metal chloride catalysts tested in this experiment all belonged to the Lewis acid category, which could meet the acidic environment required for the hydrolysis reaction of MCC (Matsumiya 2015). But the yields of the target product after catalysis were different. As the transitional metals $\mathrm{Cr}$ and $\mathrm{Sn}$ have many empty orbits, the solitary pair electrons on oxygen atoms in MCC-hydroxyl group could enter the empty orbit of metal ions and form intermediate complexes with DESs through coordination. The complex thus formed can split and recombine with MCC hydroxyl group to form proton donor, which would make glucose isomerize into fructose and followed by dehydration into the target product 5-HMF (McClure et al. 1993). However, for $\mathrm{Fe}$ and $\mathrm{Cu}$, they contain less empty orbitals than $\mathrm{Cr}$ and $\mathrm{Sn}$, so the number of lone pair electrons of oxygen atoms in cellulose hydroxyl entering into empty orbitals is small, so the ability to destroy cellulose hydrogen bond is weak, so the catalytic effect is not as good as $\mathrm{Cr}$ and $\mathrm{Sn}$.

\section{Effect of Catalyst Dosage on Product Yields}

The MCC was added to O-DES solvent at the ratio of 1:25 (mass ratio). The effect of various dosages of $\mathrm{SnCl}_{4}$ catalyst on products yields was studied at $150{ }^{\circ} \mathrm{C}$. Table 3 shows that with the increase of $\mathrm{SnCl}_{4}$ dosage, the yields of 5-HMF and glucose were 
increased first, then they remained stable, and they finally decreased. In the absence of the catalyst, the yield of 5-HMF was $6.68 \%$, indicating that the solvent system itself exhibited some catalytic activity for MCC, but the catalytic effect was poor. As the catalyst dosage increased, the yields of 5-HMF and glucose continued to increase. When the amount of catalyst reached $1.42 \mathrm{wt} \%$, the yield of 5-HMF was maximum $(8.06 \%)$, and it continued to increase with the catalyst dosage. The yields of both products decreased when the catalyst dosage was less than $1.42 \mathrm{wt} \%$. This was because of the acidity of the reaction system that did not reach the acidity level required for the reaction itself, and hence a good catalytic effect was not obtained. So the product yield continued to increase with the continuous addition of the catalyst. When the catalyst dosage was slightly more than 1.42 $\mathrm{wt} \%$, the Lewis acid concentration of the reaction system was improved, which enhanced the ability to break the MCC-glycosidic bonds. Therefore, the hydrolysis rate of MCC became faster, and the yields of glucose and 5-HMF increased. When the catalyst was excessive, the excessive acidity led to the decomposition of 5-HMF into humins, levulinic acid, and other by-products (Huddleston et al. 1998), which further reduced the yield of 5HMF.

Table 3. Effect of the Amount of Catalyst on Product Yields

\begin{tabular}{|c|c|c|c|c|c|c|c|}
\hline $\begin{array}{c}\text { Product } \\
\text { Yield (\%) }\end{array}$ & \multicolumn{7}{|c|}{ Amount of catalyst (wt\%) } \\
\cline { 2 - 8 } & 0.00 & 0.71 & 1.42 & 2.13 & 2.84 & 3.55 & 4.26 \\
\hline $5-$ HMF & 6.68 & 7.31 & 8.06 & 8.05 & 8.04 & 7.95 & 7.65 \\
\hline Glucose & 25.00 & 26.78 & 28.50 & 28.53 & 28.50 & 28.21 & 27.85 \\
\hline
\end{tabular}

\section{Effect of the Ratio of DESs to Cellulose on Product Yields}

The effect of the ratio of DESs to MCC on the yields of glucose and 5-HMF was studied at the $\mathrm{SnCl}_{4}$ dosage of $1.42 \mathrm{wt} \%$ and temperature of $140{ }^{\circ} \mathrm{C}$, as shown in Table 4 .

Table 4. Effect of the Mass Ratio of DES to Cellulose on Product Yields

\begin{tabular}{|c|c|c|c|c|c|c|c|}
\hline \multirow{2}{*}{$\begin{array}{c}\text { Products } \\
\text { Yields } \\
(\%)\end{array}$} & \multicolumn{7}{|c|}{ Ratio of DESs to cellulose } \\
\hline & 5.00 & 10.00 & 15.00 & 20.00 & 25.00 & 30.00 & 35.00 \\
\hline 5-HMF & 5.00 & 5.96 & 6.31 & 6.96 & 8.16 & 8.09 & 8.05 \\
\hline Glucose & 9.78 & 13.02 & 17.55 & 18.78 & 18.93 & 18.98 & 19.01 \\
\hline
\end{tabular}

As the amount of O-DES increased, the yields of glucose and 5-HMF also increased. When the ratio of O-DES to MCC reached 25:1, the yields of glucose and 5HMF were at $18.9 \%$ and $8.2 \%$, respectively. As the mass ratio of O-DES to MCC increased continuously, the yield of 5-HMF remained basically unchanged, and the rate of glucose formation decreased. When the ratio of O-DES to cellulose reached 35:1, the yield of glucose was only $19 \%$, the rate remained basically unchanged. The reason why the target products first remained unchanged after the increase was that DES could increase the hydrolysis rate and hydrolysis efficiency of MCC. As the amount of DES increased, the hydrogen bond network of MCC was destroyed more and more, which led to increased glucose yield, and the yield of 5-HMF also increased. When DES was added in excess, the solvent was increased, the amount of the catalyst was unchanged, and the acidity of the reaction system became low. Thus, the hydrolysis of MCC and glucose was more difficult, 
and the glucose yield was slightly decreased. On the other hand, if the generated 5-HMF was not separated in time and it can further converted into other substances, resulting in a decrease in 5-HMF yield.

\section{Effect of Reaction Temperature on Product Yields}

At a specific amount of solvent and MCC, at DES:MCC ratio of 25:1 (mass ratio), and with $1.42 \% \mathrm{SnCl}_{4}$, the effects of temperature and time on 5-HMF and glucose yields were investigated at $140{ }^{\circ} \mathrm{C}, 150^{\circ} \mathrm{C}, 160^{\circ} \mathrm{C}$, and $170{ }^{\circ} \mathrm{C}$, respectively. The experimental results were shown in Fig. 1.

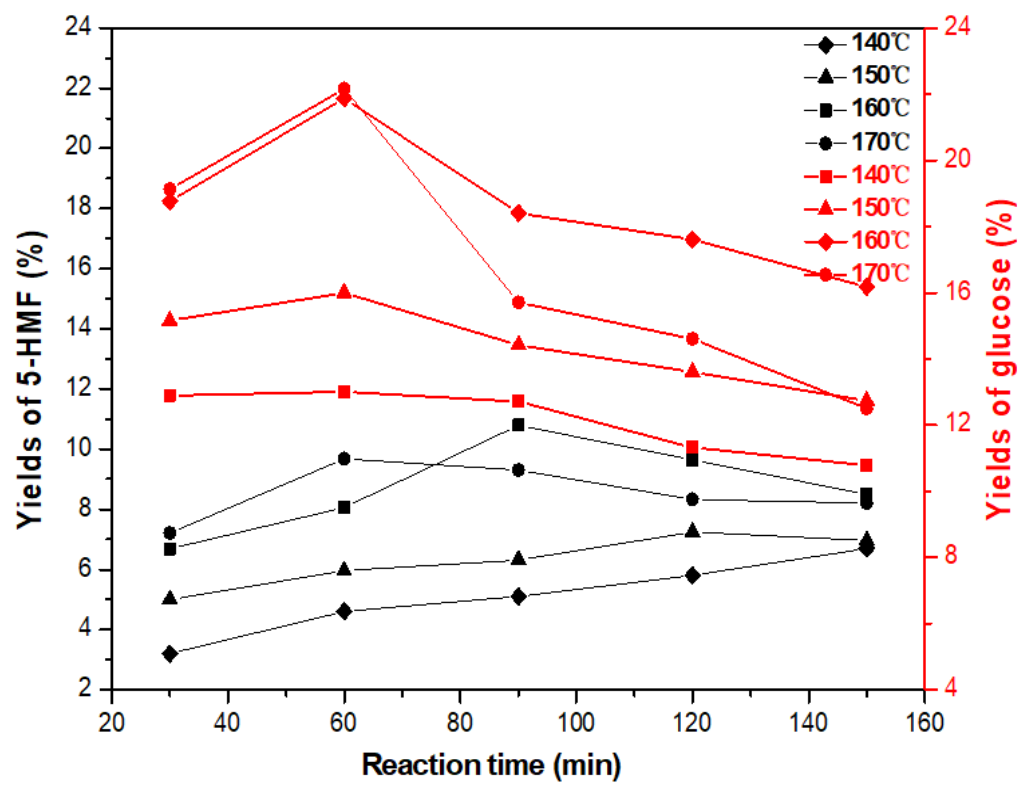

Fig. 1. Effect of reaction time and temperature on 5-HMF and glucose yields

With the increase of temperature, the yields of 5-HMF and glucose both increased first and then decreased. As the temperature was increased, the time required for 5-HMF and glucose to reach the highest yields were getting shorter and shorter. When the temperature was $140{ }^{\circ} \mathrm{C}$ and the reaction time was $30 \mathrm{~min}$, the yield of 5-HMF was $3.2 \%$. As the reaction progressed, the yield of 5-HMF increased. The yield of 5-HMF increased as the reaction progressed up to $150 \mathrm{~min}$. Thus, the temperature was not high enough to reach the temperature required for the reaction. When the temperature continued to rise, the yield of 5-HMF increased extensively. When the temperature reached $160{ }^{\circ} \mathrm{C}$, the maximum yield of 5-HMF reached at $90 \mathrm{~min}$, which was $10.79 \%$. When the temperature was continuously increased, the time at which the highest yield of 5-HMF obtained was significantly shortened, but the highest yield was $9.68 \%$, which was lower than the maximum yield of 5-HMF at $160{ }^{\circ} \mathrm{C}$. Similarly, at $140{ }^{\circ} \mathrm{C}$, the maximum yield of glucose was $13 \%$ at $60 \mathrm{~min}$, and at $160{ }^{\circ} \mathrm{C}$ and $170{ }^{\circ} \mathrm{C}$, the reaction time was $60 \mathrm{~min}$, the yield of glucose reached the maximum and remained the same.

The yields of 5-HMF and glucose increased first and then decreased with increasing temperature. This was because as the temperature increased, the movement of MCC molecules became more intense, the probability of collision increased, and the breakage of intermolecular and intramolecular glycosidic and hydrogen bonds of MCC also increased. Therefore, the rate of hydrolysis of MCC to glucose was improved, the time to the highest 
yield was shortened, the yield of glucose became higher, and the yield of 5-HMF was also increased. However, when the temperature rose to $170^{\circ} \mathrm{C}$, the yields of 5-HMF and glucose was decreased. This was because the retention of 5-HMF in the solvent was lowered at temperatures above $170{ }^{\circ} \mathrm{C}$ ( $\mathrm{Yu}$ and Tsang 2017), and 5-HMF was further hydrolyzed to by-products such as levulinic acid. In summary, when the reaction time was 90 min and the reaction temperature was $160{ }^{\circ} \mathrm{C}$, the maximum yields of glucose and 5-HMF were obtained, which were $22 \%$ and $11 \%$, respectively.

\section{CONCLUSIONS}

1. Four deep eutectic solvents (DESs) were synthesized and used to degrade microcrystalline cellulose (MCC) to prepare glucose and 5-hydroxymethylfurfural (5HMF). Among four DESs and four chloride catalysts studied, a combination of oxalic acid and choline chloride (O-DES) and $\mathrm{SnCl}_{4}$ showed the best effect on the degradation of MCC to prepare 5-HMF and glucose. Experiments had shown that the reaction temperature, reaction time, and ratio of MCC to DESs all affected the product yield. When the MCC:O-DES ratio was 1:25 (mass ratio) and $1.42 \% \mathrm{SnCl}_{4}$, at $160{ }^{\circ} \mathrm{C}$ for 90 min, the yields of 5-HMF and glucose achieved were maximum, and the yields were $11 \%$ and $22 \%$, respectively.

2. The results showed that it was feasible to use DESs as a solvent to catalyze the degradation of MCC with metal chloride as catalyst to prepare the high value-added biomass-derived chemical 5-HMF, which provided an important theoretical basis and practical application for the conservation and utilization of resources.

\section{ACKNOWLEDGMENTS}

This work was supported by the Shandong Provincial Natural Science Foundation of China (Grant No. ZR2017MC032), the Open Fund of Guangxi Key Laboratory of Polysaccharide Materials and Modification (Grant No. GXPSMM18YB-03), the Shandong Provincial Key Research and Development Program (SPKR\&DP) (Grant No. 2019GGX102029) and the Foundation of Key Laboratory of Biomass Chemical Engineering of Ministry of Education, Zhejiang University (Grant No. 2018BCE005).

\section{REFERENCES CITED}

Abbott, A. P., Capper, G., and Davies, D. L. (2003). "Novel solvent properties of choline chloride/urea mixtures," Chem. Commun. 1(1), 70-71. DOI: 10.1039/B210714G

Abbott, A. P. and McKenzie, K. J. (2006). "Application of ionic liquids to the electrodeposition of metals," Phys. Chem. Chem. Phys. 8(37), 4265-4279. DOI: 10.1039/b607329h

Bahadori, L., Abdul Manan, N. S., Chakrabarti, M. H., Hashim, M. A., Mjalli, F. S., AlNashef, I. M., Hussain, M. A., and John Low, C. T. (2013). "The electrochemical behaviour of ferrocene in deep eutectic solvents based on quaternary ammonium and phosphonium salts," Phys. Chem. Chem. Phys. 15(5), 1707-1714. DOI: 


\section{$10.1039 / \mathrm{C} 2 \mathrm{CP} 43077 \mathrm{~K}$}

Bozell, J. J., and Petersen, G. R. (2010). "Technology development for the production of biobased products from biorefinery carbohydrates - the US Department of Energy's “Top 10" revisited," Green Chem. 12(4), 539-554. DOI: 10.1039/b922014c

Casanova, O., and Iborra, S. (2010). "Biomass into chemicals: aerobic oxidation of 5hydroxymethyl-2-furfural into 2,5-furandicarboxylic acid with gold nanoparticle catalysts," ChemSusChem 2(12), 1138-1144. DOI: 10.1002/cssc.200900137

Cui, Q., Peng, X., Yao, X. H., Wei, Z. F., Luo, M., and Zu, Y. G. (2015). 'Deep eutectic solvent-based microwave-assisted extraction of genistin, genistein and apigenin from pigeon pea roots," Sep. Purif. Technol. 150, 63-72. DOI:

10.1016/j.seppur.2015.06.026

Dai, Y. T., Van Spronsen, J., Witkamp, G. J., Verpoorte, R., and Choi, Y. H. (2013). "Natural deep eutectic solvents as new potential media for green technology," Anal. Chim. Acta 766, 61-68. DOI: 10.1016/j.aca.2012.12.019

Durand, E., Lecomte, J., and Villeneuve, P. (2013). "Deep eutectic solvents: Synthesis, application, and focus on lipase-catalyzed reactions," Eur. J. Lipid Sci. Technol. 115(4), 379-385. DOI: 10.1002/ejlt.201200416

Feng, R. S., Zhao, D. B., and Guo, Y. J. (2010). "Revisiting characteristics of ionic liquids: a review for further application development," J. Environ Prot. 1, 95-104. DOI: $10.4236 /$ jep.2010.12012

Florindo, C., Oliveira, F. S., Rebelo, L. P. N., and Fernandes, A. M. (2014). "Insights into the synthesis and properties of deep eutectic solvents based on cholinium chloride and carboxylic acids," ACS Sustain. Chem. Eng. 2(10), 2416-2425. DOI: $10.1021 / \mathrm{sc} 500439 \mathrm{w}$

Ge, X., Xu, F., and Li, Y. (2016). "Solid-state anaerobic digestion of lignocellulosic biomass: Recent progress and perspectives," Bioresource Technology 205, 239-249. DOI:10.1016/j.biortech.2016.01.050.

Guo, W., Hou, Y., Wu, W., Ren, S. H., Tian, S. D., and Marsh, K. N. (2013). "Separation of phenol from model oils with quaternary ammonium salts via forming deep eutectic solvents," Green Chem 15(1), 226-229. DOI: 10.1039/C2GC36602A

Hou, X. D., Feng, G. J., Ye, M., Huang, C. M., and Zhang, Y. (2017). "Significantly enhanced enzymatic hydrolysis of rice straw via a high-performance two-stage deep eutectic solvents synergistic pretreatment," Bioresource Technol. 238, 139-146. DOI: 10.1016/j.biortech.2017.04.027

Hronec, M., Fulajtárova, K., Liptaj, T., Štolcová, M., Prónayová, N., and Soták, T. (2014). "Cyclopentanone: A raw material for production of $\mathrm{C}_{15}$ and $\mathrm{C}_{17}$ fuel precursors," Biomass Bioenerg. 63(7), 291-299. DOI:

10.1016/j.biombioe.2014.02.025

Huang, Y. B., Yang, Z., Dai, J. J., and Guo, Q. X. (2012). "Production of high quality fuels from lignocellulose-derived chemicals: a convenient $\mathrm{C}-\mathrm{C}$ bond formation of furfural,5-methylfurfural and aromatic aldehyde," RSC Advance 2(30), 11211-11214. DOI: $10.1039 / \mathrm{C} 2 \mathrm{RA} 22008 \mathrm{C}$

Huang, Y., Shen, F., La, J., Luo, G. X., Lai, J. L., Liu, C. S., and Chu, G. (2013). "Synthesis and characterization of $\mathrm{CuCl}$ nanoparticles in deep eutectic solvents," Particul. Sci. Technol. 31(1), 81-84. DOI: 10.1080/02726351.2011.648823

Huddleston, J. G., Willauer, H. D., Swatloski, R. P., Visser, A. E., and Rogers, R. D. (1998). "Room temperature ionic liquids as novel media for 'clean' liquid-liquid extraction," Chem. Commun. 1765-1766. DOI: 10.1039/A803999B 
Hu, S. Q., Zhang, Z. F., Zhou, Y. X., Song, J. L., Fan, H. L., and Han, B. X., (2009). "Direct conversion of inulin to 5-hydroxymethylfurfural in bio-renewable ionic liquids," Green Chem. 11(6), 873-877. DOI: 10.1039/B822328A

Ilgen, F., Ott, D., Kralisch, D., Reil, C., Palmberger, A., and König, B. (2009). "Conversion of carbohydrates into 5-hydroxymethylfurfural in highly concentrated low melting mixtures," Green Chem. 11, 1948-1954. DOI: 10.1039/b917548m

Liu, F., Audemar, M., Vigier, K., Cartigny, D., Clacens, J. M., Gomes, F. M. C., Pádua, A. A. H., Campo, F. D., and Jérôme, F. (2013). "Selectivity enhancement in the aqueous acid-catalyzed conversion of glucose to 5-hydroxymethylfurfural induced by choline chloride," Green Chem. 15(11), 3205-3213. DOI: 10.1039/C3GC41495G

Matsumiya, H. (2015). "Conversion of glucose into 5-hydroxymethylfurfural with boric acid in molten mixtures of choline salts and carboxylic acids," Biomass Bioenerg. 72, 227-232. DOI: 10.1016/j.biombioe.2014.11.001

McClure, P. J., Baranyi, J., and Boogard, E. (1993). "A predictive model for the combined effect of $\mathrm{pH}$, sodium chloride and storage temperature on the growth of Bronchothrix thermosphacta," International Journal of Food Microbiology. 19(3), 161-178. DOI: 10.1016/0168-1605(93)90074-Q

Mota-Morales, J. D., Gutierrez, M. C., Ferrer, M. L., Jiménez, R., Santiago, P., Sanchez, I. S., and Terrones, M. (2013). "Synthesis of macroporous poly(acrylic acid)-carbon nanotube composites by frontal polymerization in deep-eutectic solvents," J. Mater. Chem. A 1(12), 3970-3976. DOI: 10.1039/C3TA01020A

Ramesh, S., Shanti R., and Morris, E. (2013). "Characterization of conducting cellulose acetate based polymer electrolytes doped with "green" ionic mixture," Carbohydr. Polym. 91(1), 14-21. DOI: 10.1016/j.carbpol.2012.07.061

Román-Leshkov, Y., Barrett, C. J., Liu, Z. Y., and Dumesic, J. A. (2007).

"Production of dimethylfuran for liquid fuels from biomass-derived carbohydrates," Nature 447(7147), 982-985. DOI: 10.1038/nature05923

Sanchez-Leija, R. J., Pojman, J. A., and Luna-Barcenas, G. (2014). "Controlled release of lidocaine hydrochloride from polymerized drug-based deep-eutectic solvents," $J$. Mater. Chem. B 2(43), 7495-7501.

Tong, X. L., Ma, Y., and Li, Y. D. (2010). "Biomass into chemicals: Conversion of sugars to furan derivatives by catalytic processes," Applied Catalysis A: General 385(1-2), 1-13. DOI: 10.1016/j.apcata.2010.06.049

Villa, A., Schiavoni, M., Campisi, S., and Veith, G. M. (2013). "Pd-modified Au on carbon as an effective and durable catalyst for the direct oxidation of HMF to 2,5furandicarboxylic acid," ChemSusChem 6(4), 609-612. DOI: $10.1002 /$ cssc. 201200778

Wang, H. Y., Zhu, C. H., Li, D., Liu, Q. Y., Tan, J., Wang, C. J., Cai, C. L., and Ma, L. G. (2019). "Recent advances in catalytic conversion of biomass to 5- hydroxymethylfurfural and 2, 5-dimethylfuran," Renew. Sust. Energ. Rev. 103, 227-247. DOI: 10.1016/j.rser.2018.12.010

Wei, Z. F., Qi, X. L., Li, T. T., Luo, M., Wang, W., Zu, Y. G., and Fu, Y. J. (2015). "Application of natural deep eutectic solvents for extraction and determination of phenolics in Cajanus cajan leaves by ultra performance liquid chromatography," Sep Purif Technol 149, 237-244. DOI: 10.1016/j.seppur.2015.05.015

Yu, I. K. M., and Tsang, D. C. W. (2017). "Conversion of biomass to hydroxymethylfurfural: A review of catalytic systems and underlying mechanisms," Bioresource Technol. 238, 716-732. DOI: 10.1016/j.biortech.2017.04.026 
Zhang, H., Wang, Z., and Gao, H. K. (2018). "Preparation of 5-hydroxymethylfurfural based on the biphasic system of ionic liquid/ethyl butyrate," BioResources 13(1), 1189-1201. DOI:10.15376/biores.13.1.1189-1201.

Zhao, H., Baker, G. A., and Holmes, S. (2011). "Protease activation in glycerol-based deep eutectic solvents," J. Mol. Catal. B-Enzym. 72(3-4), 163-167. DOI: 10.1016/j.molcatb.2011.05.015

Zuo, M., Le, K., and Jiang, Y. T. (2017). "Green process for production of 5hydroxymethylfurfural from carbohydrates with high purity in deep eutectic solvents," Industrial Crops \& Products. 99, 1-6. DOI:10.1016/j.indcrop.2017.01.027.

Article submitted: December 7, 2019; Peer review completed: February 29, 2020;

Revised version received and accepted: March 13, 2020; Published: March 25, 2020.

DOI: 10.15376/biores.15.2.3344-3355 


\section{SUPPLEMENTARY APPENDIX}

\section{Preparation of DESs}

The required reagents were dried in a vacuum drying chamber at $50{ }^{\circ} \mathrm{C}$ for $48 \mathrm{~h}$. Hydrogen bond donors and hydrogen bond acceptors (molar ratio=2:1) were added to a four-neck flask, stirred and heated under nitrogen in an oil bath at certain temperature. When a fully homogeneous transparent liquid was obtained, the reaction was stopped, and transferred to a plug bottle. Then, the prepared DESs were dried for $48 \mathrm{~h}$ in a vacuum dryer (vacuum dryer, DHG, Ta Company, USA) at $70^{\circ} \mathrm{C}$ and stored in a desiccator. The synthesis conditions of four DESs are listed in Table 1.

Table S1. Synthesis Conditions of Four DESs

\begin{tabular}{|c|c|c|c|c|c|}
\hline \multirow[b]{2}{*}{ HBA } & \multirow[b]{2}{*}{ HBD } & \multicolumn{3}{|c|}{ Synthesis conditions } & \multirow{2}{*}{$\begin{array}{c}\text { Types o } \\
\text { DESs }\end{array}$} \\
\hline & & Molar ratio(HBA:HBD) & $\begin{array}{c}\text { Temperature } \\
\left({ }^{\circ} \mathrm{C}\right)\end{array}$ & $\begin{array}{l}\text { Time } \\
(\mathrm{min})\end{array}$ & \\
\hline \multirow{4}{*}{$\begin{array}{l}\text { Choline } \\
\text { chloride }\end{array}$} & Citric acid & \multirow{4}{*}{$1: 2$} & 80 & 60 & C-DES \\
\hline & Glycerol & & 35 & 10 & G-DES \\
\hline & Urea & & 80 & 120 & U-DES \\
\hline & Oxalic acid & & 50 & 30 & O-DES \\
\hline
\end{tabular}

The DESs synthesized with choline chloride as hydrogen bond acceptor and oxalic acid, citric acid, urea, and glycerol as hydrogen bond donors were abbreviated as O-DES, C-DES, U-DES, and G-DES, respectively.

\section{Effect of Temperature on the Viscosity of DESs}

It can be seen from Fig. S1 that the viscosity of DESs is sensitive to temperature change. With the increase of temperature, the viscosity of DESs decreased, because the effects of the van der Waals force and hydrogen bond force in the system are weakened gradually. At the same temperature, the viscosity of U-DES was significantly higher than that of the other three DESs, followed by G-DEs and C-DES, and O-DES had the smallest viscosity.

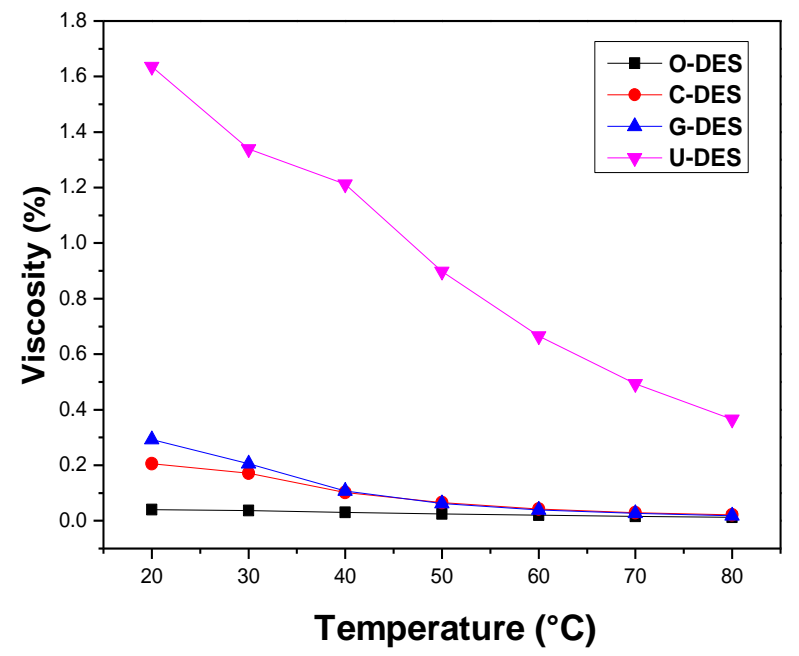

Fig. S1. Effect of temperature on the viscosity of DESs 
Low viscosity is beneficial to the mass transfer between solute and solvent and provides higher permeability into cellulosic material having a tight structure. This is conducive to the formation of new molecular hydrogen bond between DESs and cellulose and increases the solubility of cellulose. When the temperature continued to rise to $40{ }^{\circ} \mathrm{C}$, the decreasing trend of DESs' viscosity was not obvious. This is because the force between DESs molecules is not sensitive to the temperature change, so the temperature has a poor effect on the viscosity. 